\author{
Elisabetta Grande \\ (professore ordinario di Diritto comparato nell'Università degli Studi del \\ Piemonte Orientale, Dipartimento di Giurisprudenza e Scienze Politiche, \\ Economiche e Sociali)
}

\title{
Il caso dell'aborto: una buccia di banana per la SCOTUS? ${ }^{*}$ *
}

The case of abortion: a slippery slope for SCOTUS?

\begin{abstract}
The case of the right of abortion in the United States is going to represent much more than a mere legal question. The upcoming decision of the Supreme Court of the United States (SCOTUS) on the matter involves its own credibility as a third party institution in a check and balance system where the SCOTUS is increasingly perceived as a political player. In this paper the A. tries to explore why it is so.
\end{abstract}

SOMMARIO: 1. La questione dell'aborto e la perdita di credibilità della Corte Suprema statunitense - 2. Una tempesta perfetta? - 3. La partita della fiducia.

\section{1 - La questione dell'aborto e la perdita di credibilità della Corte Suprema statunitense}

Mai come in questo momento la Corte Suprema statunitense sta perdendo legittimazione. È un problema serio, perché - come si è più volte scritto su queste pagine - è proprio sulla sua autorevolezza che si fonda il corrispondente potere di controllo sull'operato tanto del legislativo quanto dell'esecutivo. A differenza, infatti, del potere legislativo della borsa e di quello esecutivo della spada, che trovano fondamento esplicito nella Costituzione federale, quello della SCOTUS, quale giudice ultimo delle leggi (e dell'azione dell'esecutivo), è un potere che essa stessa si è attribuito a partire dalla nota sentenza Marbury $v$. Madison nel 1803, grazie al genio del suo Chief Justice, John Marshall. La storia ci racconta come l'accettazione di quella prerogativa in capo a una Corte che si era in tal modo posta al vertice di una piramide giudiziaria, cui dopo di allora sarebbe spettato decidere sulla costituzionalità delle leggi non solo federali ma anche statali, non sia avvenuta senza conflitti, anche pesanti. Fu

\footnotetext{
${ }^{1} \mathrm{Il}$ contributo, non sottoposto a valutazione, è stato pubblicato nella rivista telematica "Questione Giustizia" (www.questionegiustizia.it) il 27 ottobre 2021.
} 
l'autorevolezza di quei nove giudici, che non solo parlavano all'unisono (in 35 anni di gestione Marshall le opinioni concorrenti o tanto meno dissenzienti si possono contare sulle dita di una mano!), ma che soprattutto si presentavano in qualità di tecnici e non di politici, a consentire alla Corte Marshall di consolidare l'auto attribuitosi potere di così detta judicial review. La famosa counter majoritarian difficulty esplicitata nel 1962 da un professore della Yale Law School, Alexander Bickel, che si domandava in forza di quale principio nove giudici, nominati in via indiretta, siano legittimati a invalidare le leggi emanate dai tanti, eletti invece in via diretta, che rappresentano il volere della maggioranza dei cittadini ${ }^{2}$ - può infatti essere risolta solo attraverso un'immagine di assoluta neutralità politica della Corte, che sia percepita dall'opinione pubblica come bocca della carta costituzionale voluta dal popolo statunitense. Decisioni il più possibile unitarie (agevolate dall'invenzione di Marshall dell' „opinione della Corte", in luogo delle tante opinioni fornite per esempio dai giudici inglesi), una nomina dei nove giudici supremi sganciata da divisioni partitiche, oltre all'emanazione di sentenze equilibrate, giuridicamente argomentate a fondo e il più possibile in sintonia con il sentimento collettivo - quando l'inevitabile evoluzione del diritto comporta un révirement giurisprudenziale - rappresentano da sempre gli ingredienti di quella legittimazione.

Quei fattori chiave, indispensabili per la credibilità di una Corte che si è auto attribuita il più penetrante fra i poteri - quello di controllo degli altri due - sembrano oggi venuti meno. Il colpo di grazia è certamente arrivato con la decisione del primo settembre 2021, con cui la SCOTUS ha respinto con una maggioranza di 5 a 4 il ricorso contro la legge texana, che vieta sempre l'aborto oltre la sesta settimana di gravidanza ${ }^{3}$, presentato dalle cliniche del Texas per ottenerne la sospensiva. Il breve comunicato con il quale la Corte ha mantenuto in vita una legge - per la prima volta costruita in modo tale da essere attaccata con difficoltà prima della sua entrata in vigore - che stabilisce una ricompensa di 10.000 dollari (a titolo di risarcimento di danno presunto) per chiunque intenti una causa contro chi abbia praticato o agevolato un aborto fuori dai limiti consentiti (una vera e propria taglia da profondo Far West in capo ai violatori della

2 A. BICKEL, The Least Dangerous Branch. The Supreme Court at the Bar of Politics, Yale U. P., 1986

3 A partire dal momento in cui è verificabile un'attività cardiaca del feto, dice precisamente il Texas Senate Bill 8. 
norma! $)^{4}$, ha buttato molti nello sgomento. Certo si è trattato di una decisione interlocutoria, in attesa della presa di posizione sul merito della questione con la pronuncia su un'altra legge, quella del Mississippi, che impedisce l'esercizio del diritto di aborto dopo la $15^{\text {ma }}$ settimana, ossia circa due mesi prima del momento indicato dalle sentenze Roe v. Wade (1973) e Planned Parenthood v. Casey (1992). In tali casi la Corte, si sa, aveva chiarito che in forza del XIV emendamento della Costituzione federale nessuno Stato può imporre un "undue burden", ossia un onere eccessivo, alla donna che scelga di interrompere la sua gravidanza prima della "viability" del feto - ossia prima del momento in cui quest'ultimo sia capace di vita autonoma. La sentenza sulla costituzionalità della normativa del Mississippi, attualmente dichiarata illegittima dalle corti inferiori, è in calendario per la sua prima udienza il primo dicembre di quest'anno $^{5}$. La decisione della SCOTUS di decidere nuovamente in tema di interruzione volontaria di gravidanza e di prendere di fronte a sé il caso del Mississippi - avendo la stessa piena discrezionalità nella selezione delle questioni su cui interloquire in ultima istanza - è segno che l'organo supremo vuole ancora dire la sua in proposito. A differenza dei più recenti casi su cui la Corte si è pronunciata - relativi a quali medici potessero praticare l'aborto o quali standard minimi dovesse rispettare la clinica abortiva o ancora quali metodi fossero accettabili - si tratterà questa volta di una presa di posizione che andrà al cuore della questione. Il tema specifico sul quale i supremi giudici si pronunceranno è: «se prima della "viability" qualunque divieto di interruzione volontaria di gravidanza sia incostituzionale». A seconda della risposta che darà, la Corte riaffermerà, rivedrà $\mathrm{o}$ rovescerà i suoi precedenti. Quale strada i supremi giudici imboccheranno è tutto da vedere, ma la mancata sospensiva della legge texana, che - dopo l'ulteriore via libera ottenuto dalla corte d'appello federale del quinto circuito, pronunciatasi in controtendenza rispetto al ricorso vittoriosamente presentato in prima istanza dal dipartimento di giustizia statunitense ${ }^{6}$ - ha di fatto cancellato il diritto di abortire delle donne di quello Stato, non rappresenta certo una spia incoraggiante per chi ritiene che Roe $v$. Wade, così come interpretato in Planned Parenthood $v$.

${ }^{4}$ Per approfondimenti mi permetto di rinviare al mio Stati Uniti, la Corte Suprema cancella provvisoriamente il diritto di abortire delle donne texane (al sito https://www.micro mega.net/corte-suprema-aborto-texas/).

${ }^{5}$ Il caso è Dobbs v. Jackson Women's Health Organization, No. 19-1392.

${ }^{6}$ https://www.nytimes.com/2021/10/14/us/politics/texas-abortion-appeals-court.htm 
Casey, costituisca un punto di non ritorno sulla strada della libertà delle donne.

\section{2 - Una tempesta perfetta?}

È proprio la pronuncia del primo settembre, d'altronde, ad avere fatto esplodere il malcontento nei confronti dell'organo autoproclamatosi onnipotente, la cui legittimazione a porre nel nulla le leggi, tanto della federazione che degli Stati, appare oggi in grande crisi. Se, infatti, già la nomina di Amy Coney Barrett nell'ottobre dell'anno scorso aveva inferto un duro colpo alla sua immagine di neutralità per le modalità e i tempi con cui essa era avvenuta ${ }^{7}$, con quella decisione è apparso chiaro come tutti e tre gli elementi necessari per garantire l'apoliticità alla Corte Suprema siano venuti definitivamente meno.

Non è stata soltanto l'assenza di una presa di posizione unitaria da parte della SCOTUS ad averne abbattuto la credibilità come organo tecnico, laddove la frammentazione al suo interno non avrebbe potuto essere più estrema, nonostante il tentativo del Chief Justice Roberts di evitare una divisione su linee puramente partitiche. La SCOTUS ha, infatti, da tempo abituato l'opinione pubblica statunitense a decisioni non unitarie. È stato piuttosto il troppo evidente allineamento dei giudici conservatori alle posizioni del partito dal quale sono stati nominati sulla scorta di una polarizzazione del tutto inedita, unito alla mancanza di motivazioni in punto di diritto - tipica delle pronunce del cosiddetto "shadow docket" della Corte - e alla siderale distanza rispetto al sentimento collettivo della posizione espressa con il suo provvedimento, che peraltro rovescia apertamente la propria precedente linea giurisprudenziale, ad avere creato le condizioni per la tempesta perfetta.

La decisione di non sospendere in via emergenziale l'efficacia della legge texana anti aborto è stata, infatti, sostenuta da 5 giudici conservatori percepiti come politici più che come tecnici, perché nominati da un Senato diviso su linee partitiche come non era avvenuto mai prima. Se un presidente di minoranza come Trump ha potuto nominare con i voti dei soli senatori repubblicani (o quasi) - anch'essi peraltro espressione della minoranza e non della maggioranza del paese - Amy Coney Barrett (52 a 48), Brett Kavanough (50 a 48) e Neil Gorsuch (54 a 45), ugualmente con

\footnotetext{
7 Mi permetto di rinviare al mio Amy Coney Barrett nel dilemma democratico (al sito: https://www.questionegiustizia.it/articolo/amy-coney-barrett-nel-dilemma-democratico).
} 
pochissimi voti dei senatori democratici furono investiti della carica a giudice supremo anche gli altri due giudici che a settembre si sono espressi contro la sospensiva, ossia Samuel Alito (58 a 42) e Clarence Thomas (52 a 48), rispettivamente nominati da Bush figlio e padre nel 2005 e nel 1991. Entrambi, così come per ora i tre Justices nominati da Trump, non hanno mai seriamente scartato rispetto a posizioni fortemente conservatrici, confermando implicitamente la linea politica della loro nomina. Si tratta di un quadro assai differente rispetto a quanto è avvenuto nel passato. Si pensi soltanto all'unanimità al Senato o alle maggioranze assai vicine a essa con cui furono investiti della carica Anthony Kennedy (97 a 0), Ruth Ginzburg (96 a 3), Antonin Scalia (98 a 2), Sandra O'Connor (99 a 0), Paul Stevens (98 a 0), o David Souter (90 a 9), solo per fare qualche esempio, al di là che a nominarli fosse un presidente repubblicano - come nel caso di tutti coloro che ho indicato salvo Ruth Bader Ginzburg - o meno. Si pensi d'altronde a quanto nel passato i supremi giudici, pur scelti da presidenti repubblicani, abbiano poi assunto posizioni liberali e progressiste, come Stevens o Souter, o si siano collocati a metà strada, a volte votando con i liberali a volte con i conservatori, come la $\mathrm{O}^{\prime}$ Connor o Kennedy. Non c'è dunque da stupirsi se, pur a fronte di una decisione altamente opinabile in termini tecnici, quale fu quella con cui nel 2000 - con Bush v. Gore - la SCOTUS attribuì di autorità la presidenza a George W. Bush, essa non perse di credibilità come sembra averne persa oggi. A differenza di allora i 5 giudici conservatori della SCOTUS odierna sono, infatti, percepiti come contemporaneamente espressione di una sola parte politica e indissolubilmente a essa allineati.

La pronuncia della Corte del primo settembre, inoltre, non soltanto si pone in pieno contrasto con la sua giurisprudenza precedente, che dal 1973 in poi ha tutelato la scelta della donna di interrompere la propria gravidanza fino a un momento assai avanzato, in quanto espressione della sua autodeterminazione come tale protetta dal XIV emendamento della Costituzione ("The right of privacy [...] is broad enough to encompass a woman's decision to terminate her pregnancy"8). Essa è altresì in totale divergenza rispetto al sentimento collettivo, come dimostrano tre sondaggi condotti poco dopo il primo settembre, da cui risulta che meno di un terzo degli americani sono favorevoli alla sovversione di Roe $v$. Wade . Ma non basta. Si tratta anche di una pronuncia emessa senza che la

${ }^{8}$ Roe Et Al. V. Wade, District Attorney Of Dallas County, No. 70-18, Supreme Court Of The United States, 410 U.S. 113, January 22, 1973.

${ }^{9}$ https://edition.cnn.com/2021/09/22/politics/supreme-court-polling-roe-wade/index.html 
Corte abbia ascoltato difese orali o letto memorie scritte, priva tanto di motivazioni quanto di firma del suo autore. È una decisione parte di quegli orders annunciati mezz'ora prima delle udienze in cui vengono discusse nel merito le cause, cui la dottrina ha dato il nome significativamente sinistro di "shadow docket", ossia di decisioni del registro ombra ${ }^{10}$. Sono ingiunzioni o sospensive emergenziali il cui eccessivo uso è da tempo criticato, perché si tratta di pronunce poco trasparenti sotto il profilo tanto delle ragioni che sottendono le scelte effettuate, quanto sotto quello della responsabilità dei singoli giudici in relazione alla decisione adottata, giacché spesso essi non esprimono il loro assenso o dissenso. Come scrive Elena Kagan, uno dei Justices progressisti della SCOTUS che ha votato con la minoranza, il caso della legge del Texas sull'aborto

\begin{abstract}
"illustra proprio quanto le pronunce dello shadow docket della Corte possano grandemente allontanarsi dagli ordinari principi dei processi di impugnazione" ed "è emblematico dell'uso eccessivo di simili decisioni, che ogni giorno che passa diventano sempre più prive di motivazione, incoerenti e impossibili da difendere"11.
\end{abstract}

\title{
3 - La partita della fiducia
}

Sono questi gli esplosivi ingredienti della tempesta perfetta che si sta oggi abbattendo sulla SCOTUS, la cui fiducia da parte dell'opinione pubblica è crollata drammaticamente. Un sondaggio Gallup, effettuato poco dopo il primo settembre, riporta un'approvazione dell'operato della Corte del solo $40 \%$ degli americani contro il 58\% dell'anno precedente: il tasso più basso da quando - 21 anni fa - questo tipo di sondaggio è stato effettuato ${ }^{12}$. Una diversa indagine condotta nello stesso periodo dall'Annenberg Public Policy Center dell'Università della Pennsylvania, rileva d'altronde che il $34 \%$ degli americani pensa che, se la Corte continuasse a emettere decisioni in contrasto con l'opinione della maggioranza della popolazione,

\footnotetext{
10 L'invenzione del nome shadow docket, senza intento denigratorio, sebbene critico, si deve a William Baude, che nel 2005 scrive The Supreme Court's Shadow Docket (reperibile al sito: https://chicagounbound.uchicago.edu/cgi/viewcontent.cgi?article=1961Econtext=public_law _and_legal_theory).

11 Cfr. A. LIPTAK, J.D. GOODMAN, S. TAVERNISE, Supreme Court, Breaking Silence, Won't Block Texas Abortion Law (al sito: https://www.nytimes.com/2021/09/01/us/supremecourt-texas-abortion.html).

12 https://news.gallup.com/poll/354908/approval-supreme-court-down-new-low.aspx
} 
sarebbe assai meglio farne a meno. Due anni fa solo il $20 \%$ degli americani aveva espresso un parare analogo.

Per quanto i giudici supremi cerchino di difendere le proprie decisioni come frutto di pure logiche giuridiche, accusando i loro detrattori di presentarle impropriamente come determinate da ideologie politiche ${ }^{13}$, l'impressione è che vi riescano sempre meno. Il potere che si sono auto conferiti circa 220 anni fa è oggi accettato con sempre maggiore difficoltà, tanto che da più parti si sollevano richieste di ampliare il numero dei membri della Corte Suprema e/o di ridurne temporalmente il mandato, che oggi è a vita. È per questo che il semestre in corso appare davvero cruciale per la Corte Suprema statunitense, che sulla questione aborto giocherà con ogni probabilità una partita decisiva per la propria credibilità.

13 L. GREENHOUSE, The Supreme Court's Pain, and Our Anger (al sito: https://www.nytimes.com/2021/10/07/opinion/supreme-court-polls-abortion-bush-gore.html), e A. LIPTAK, Back on the Bench, the Supreme Court Faces a Blockbuster Term (https://www.nytimes.com/2021/10/03/us/politics/supreme-court-new-term.html). 